\title{
HISTORIA
}

\section{La epidemia de cólera morbo de 1884 en Elche; un elemento de cambio de sociedad y una nueva categoría de la contemporaneidad}

\section{The cholera morbo epidemic of 1884 in Elche; An element of change of society and a new category of contemporary}

\section{A epidemia de cólera morbo de 1884 em Elche; Um elemento de troca de sociedade e uma nova categoria da contemporaneidade}

\section{Mariano Monge Juárez}

Doctor en Historia Contemporánea. Profesor de historia en la Universidad de Murcia.

Cómo citar este artículo en edición digital: Monje Juárez, M. (2018). La epidemia de cólera morbo de 1884 en Elche; un elemento de cambio de sociedad y una nueva categoría de la contemporaneidad. Cultura de los Cuidados (Edición digital),

22(51). Recuperado de < http://dx.doi.org/10.14198/cuid.2018.51.04>

Correspondencia: remitirse al correo electrónico.

Correo electrónico: monge.juarez@ua.es

Recibido: 07/12/2017; Aceptado: 17/03/2018

\begin{abstract}
The arrival of the cholera epidemic in Elche, a western Mediterranean city during 1884-85, produces a set of responses: Urban changes, improvements in public health, denunciation from the press, and, above all, the development of the pro-union associative movement. The objective of this article is to interpret these responses as a set of categories of change of society that function as biopolitical strategies and their analysis can constitute a model of hermeneutic for other objects of study.

Keywords: cholera, epidemic, public health, associationism, biopolitics
\end{abstract}

\section{RESUMEN}

La llegada de la epidemia de cólera a Elche, una ciudad del Mediterráneo occidental, durante 1884-85, produce un conjunto de respuestas:

cambios urbanísticos, mejoras en la salud pública, denuncia desde la prensa, y, sobre todo, desarrollo del movimiento asociativo protosindical. El objetivo de este artículo es interpretar estas respuestas como un conjunto de categorías de cambio de sociedad que funcionan como estrategias biopolíticas y su análisis puede constituir un modelo de hermenéutico para otros objetos de estudio.

Palabras clave: Cólera, epidemia, salud pública, asociacionismo, biopolítica.

\section{RESUMO}

A chegada da epidemia de cólera em Elche, uma cidade no Mediterrâneo ocidental durante 1884-1885, produz um conjunto de respostas: 
mudanças urbanas, melhorias na saúde pública, relatórios da imprensa e associações de desenvolvimento especialmente protosindical. $\mathrm{O}$ objetivo deste artigo é interpretar estas respostas como um conjunto de categorias de mudança da sociedade que funcionam como estratégias biopolíticas e análise pode ser um modelo hermenêutico para outros objetos de estudo.

Palavras chave: Cólera, epidemia, saúde pública, asociacionismo, biopolítica

\section{INTRODUCCIÓN OBJETIVOS}

El primer objetivo de este artículo es ofrecer un modelo de análisis que vincule la epidemia de cólera con el cambio de sociedad, es decir, pretende el estudio de las respuestas y las consecuencias económicas, sociales y políticas que genera la presencia del huésped del Gánges en una ciudad de unos veinticinco mil habitantes, situada en el Mediterráneo occidental, durante las últimas décadas siglo XIX y que, desde diversos sectores de la sociedad, han quedado registrados en diferentes documentos.

El segundo objetivo es presentar el resultado narrativo del análisis del cuerpo documental localizado en el Archivo Histórico Municipal de Elche (AHME): la prensa local, sesiones municipales, legajos u otros documentos, rastreando los significados y contenidos materiales de la epidemia de cólera.

\section{METODOLOGÍA}

El método seguido para afrontar el presente microestudio es el hipotético-deductivo, expuesto por Ciro Cardoso (Cardoso, 1989). Por tanto, tras una primera aproximación desde la bibliografía existente acerca del cólera en Europa, España, y en concreto, en la franja mediterránea, y una ubicación contextual desde el punto de vista de la historiografía general acerca de la ciudad de Elche a finales del siglo XIX, hemos enunciado una hipótesis de partida, que forma parte del análisis ejercido sobre todo el proceso de cambio de sociedad entre $1884 \mathrm{y}$ 1903, desde el zócalo infraestructural, que entendemos por los factores económicos y ecológicos, hasta los aspectos superestructurales, políticos, sociales o culturales. De este modo, situamos la epidemia de cólera en un lugar de transición entre ambas estructuras, desprendido de la base ecológica e imbricado en la cultura de la sociedad, sobre todo por sus consecuencias económicas y sociales. Este planteamiento nos ha servido para llegar a la siguiente hipótesis general: Entendemos el conjunto de respuestas sociales y políticas a la epidemia como categorías fundamentales en el cambio de sociedad hacia la contemporaneidad. La sociedad se cuidará de la epidemia y generará, a su vez, elementos nuevos para cuidar a sus enfermos, estos nuevos elementos son factores decisivos en la transición hacia la contemporaneidad.

\section{DESARROLLO DE LA EPIDEMIA DE CÓLERA MORBO}

Las epidemias son un fenómeno que combina la dialéctica global-local, es decir, se trata de hechos glocales. Durante las épocas preindustriales no es posible para los coetáneos seguir con exactitud los ciclos epidémicos, pero desde mediados del siglo XIX, gracias al desarrollo del ferrocarril, el telégrafo y la prensa, las epidemias son predecibles, lo cual conlleva cierta capacidad para idear respuestas nuevas. Uno de esos rasgos que define la contemporaneidad es precisamente la capacidad de seguir el movimiento que describe el huésped del Ganges desde lugares tan lejanos como Rusia o Nueva York, hasta poder sentir ya el olor de la muerte en los pueblos más cercanos. La presencia de la infección será recurrente, y hasta finales del siglo XX, su amenaza formará parte de la vida. Ciudades como Valencia, Alicante, Murcia, Cartagena (Sáez Gómez, 2004), Almería o Cádiz (Herrera, 1997) sufren los rigores de la enfermedad constantemente, y ofrecen soluciones similares. Después de la epidemia, la ciudad no volverá a ser la misma, sobre todo porque las respuestas organizativas a la enfermedad cambian la sociedad ilicitana de modo irreversible. Una condición fundamental es que el despegue y crecimiento de la economía de Elche -industrialización- tiene lugar en ese segmento comprendido entre dos pandemias: la del cólera, de 1884-86 y la de la gripe 1916-19, periodo en el que, además, la ciudad afronta el paludismo endémico, la peste bubónica en 1899 o la viruela, en 1902.

El cólera había llegado a la península hacia 1830. La primera epidemia reseñable en el Reino de Valenciano y en la entonces villa de Elche tiene lugar en 1834. La población ya había inaugurado el siglo XIX con la fiebre amarilla de 
1811, aunque el momento más severo, sin duda, es el cólera de 1854-55, que regresa, también con virulencia, en 1865 (Ibarra Ruiz, 1895). Los ilicitanos se acostumbran a convivir con los ciclos de la enfermedad, que golpea con especial incidencia sobre clase trabajadora, mal alimentada y en condiciones de vida propicias para la infección. Como advierte el doctor Luis Gómez, ya en la segunda década del siglo XX, la deficitaria dieta del obrero, unida a algunas costumbres poco higiénicas, como no hervir la leche de cabra antes de ser consumida, produce una lista de enfermedades frecuentes como paludismo, las fiebres de malta, la gripe, el tifus y sobre todo, la tuberculosis (Moreno, 1987). La idea de infección y de epidemia -compañera de la miseria- se imbrica en la sociedad de tal modo que hemos de hablar de toda una cultura social y política de la enfermedad: mientras las familias todavía visten el luto por sus muertos en la epidemia de septiembre y octubre de 1884, en noviembre de ese mismo año se anuncia una pieza humorística escrita por un ilicitano, se titula Los microbios, y se estrena en el teatro Recreo (El Vinalopó, 34). La epidemia es una metáfora para la peor comparación, como la que haga el periodista Ildefonso Sansano cuando en enero de 1885, equipare en sentido figurado la plaga de los conservadores con el Asiático, (El Vinalopó, 49) del que también se sirva el caciquismo local para ajustar un poco más las tuercas de su poder en la ciudad, como advierte la prensa local, y que se traduce en la ayuda de 10.000 pesetas que recibe el municipio, atribuida a la intervención del marqués del Bosch. ${ }^{1}$ Así relata un semanario local los sentimientos de un pueblo aterrorizado que busca apoyo en las redes caciquiles de la ciudad: "En aquellos aciagos días, nosotros vimos que numerosísimos grupos de trabajadores se dirigían a la casa del notario Sr. Gómez; nosotros oímos como uno de ellos en nombre de sus compañeros, le esponía el deplorable estado a que, una vez cerradas las fábricas, habían quedado reducidos todos; nosotros observamos que el Sr. Gómez despedía al Sr. Marqués del Bosch pintándole la angustiosa situación de Elche, y leímos después la contestación del Sr. Rojas condoliéndose de la desgracia tanta y anunciando que había telegrafiado al ministerio de la Gobernación para que acudiera a remediarla; todavía no habían pasado dos horas, nosotros leímos otro telegrama dirigido al Sr. Gómez por el Sr. Rojas, en que transmitía a este la contestación del ministerio prometiendo que del fondo de calamidades públicas concedería a Elche cuarenta mil reales...” (El Vinalopó, 52)

La cuestión es que el cólera es el centro de atención de ricos y pobres. Por primera vez se genera un foco de información activo, inquietud social y opinión pública en torno a la epidemia, que se observa sobre todo desde la visita del propio Jaime Ferrán en agosto de 1885, en plena controversia sobre la cuestión Ferrán, (Báguena, 2011) visita que deja una fuerte impronta en los documentos del municipio.

\section{LA LLEGADA DEL HUÉSPED DEL GÁNGES, 1884-85}

A principios de junio de 1884, El Vinalopó advierte a sus lectores que el terrible huésped del Gánges está causando víctimas en algunas de las más importantes poblaciones de la vecina república francesa, provocando el luto y el dolor en el hogar doméstico y paralizando todos los contratos y transacciones mercantiles. Ante la gravedad de los hechos, el semanario local cree oportuno adelantar las vacaciones escolares, cuidar la limpieza de cloacas y sobre todo la más escrupulosa vigilancia e inspección de todos los víveres que se expenden en el mercado público, $y$ especialmente en las frutas y carnes. (El Vinalopó, 21) La presencia del cólera en el sur de Francia reta a las autoridades. A principios de julio de 1884, inquieto por la relativa proximidad de la enfermedad, el alcalde, acompañado de los concejales Perpiñán y Bañón, los facultativos Blas Blasco y Santiago Pomares, más el periodista Ildefonso Sansano, visitan los barrios más apartados de la ciudad (El Vinalopó, 22). A su vez, el alcalde de Alicante inicia una suscripción popular, cuyo producto se invertirá en acordonar la población, si así lo exigen las circunstancias, o en socorrer a los pobres en el caso de que el cólera morbo asiático invadiese la capital. (El Vinalopó, 23)

Esa mima semana del 20 de julio, el médico Santiago Pomares Ibarra publica un artículo titulado "El Cólera” en el semanario de mayor tirada de la ciudad. Se trata de un recorrido histórico de la epidemia hasta la presencia de la enfermedad en Tolón y Marsella. En aquellos mismos momentos, pueblos franceses tan cercanos a nuestra España, lo cual hace que miremos con mucho más respeto la 
aparición del mal en nuestra ciudad. (El Vinalopó, 22) Pocos días después, un telegrama de la Dirección de Beneficencia y Sanidad asegura que la salud pública no ofrece ninguna alteración en la Península. No obstante, llega la noticia de que una embarcación procedente de Marsella se encuentra en cuarentena en Mahón, tras el fallecimiento de una de sus pasajeras y la enfermedad sospechosa de un pasajero y un tripulante. Además, se informa de los 58 muertos en la citada ciudad francesa. (El Vinalopó, 22)

El cólera se encuentra presente en Aix, Cerre, Tholne, Arles, Avignón, y Marsella, y según la "Ultima hora” del Gobierno civil, se han registrado 26 defunciones más, mientras, en España se disfruta de perfecta salud. Las informaciones son contradictorias. La confusión y el miedo crece entre la población. Los telegramas dan cuenta de que la enfermedad decrece en el sureste de Francia, pero el devenir de los hechos no tranquiliza a la prensa local, sobre todo tras la llegada a Novelda de un buen número de toneles para vino procedentes de Marsella, cuyo propietario no ha querido hacerse cargo de ellos por temor a los microbios. Desde sus páginas, El Vinalopó interpela al gobernador civil; si las referidas mercancías han sido o no recibidas en territorio español con arreglo a lo provenido a las vigentes disposiciones higiénicas dictadas recientemente por la Dirección de Sanidad. (El Vinalopó, 25)

La ciudad es consciente de la amenaza. El 10 de agosto se denuncia la existencia de aguas estancadas en el pantano, y el director general de carabineros ordena la limpieza urgente de los cuarteles. (El Vinalopó, 26) La celebración del Misteri es suspendida, y el día 31 de agosto, el cólera es oficialmente declarado en la ciudad, que pronto es acordonada. La prensa deja de publicarse. El Ayuntamiento, presidido por Andrés Tarí y los facultativos municipales capitaneados por el Dr. Campello se pone al frente de la crisis, aunque es en la práctica se el médico y filántropo Santiago Pomares Ibarra el que se enfrenta a la enfermedad en la calle, sobre todo entre los más necesitados. La epidemia permanece en Elche desde ese 31 de agosto hasta el 11 de octubre de 1884, día que se celebra en la basílica de Santa María el Te Deum laudum, significando al mundo que no mora ya en Elche el funesto huésped del Gánges, (El Vinalopó, 30) aunque el cinturón sanitario no se levanta hasta que no pasen unos días, y una semana después se alzan los de Novelda y Monforte (El Vinalopó, 30). En Elche, la cifra oficial de invadidos es de 114, de los que fallecen 67, (El Vinalopó, 30) sobre una población de unos 18.000 habitantes, de los cuales, la mitad viven en el casco urbano, donde precisamente incide la enfermedad.

Una vez superada la fase de 1884, de nuevo la prensa -liderada por Ildefonso Sansanoinicia una campaña informativa y crítica; primero reconstruye paso a paso los hechos de la epidemia, y segundo, lanza una andanada de críticas contra el Ayuntamiento, el Gobierno civil e incluso el Obispado.

Pero el mal asiático queda en estado latente. A partir de julio de 1885, vuelve a ser el tema esencial de la prensa. El Bou advierte de nuevo que mos se aproxima a pasos agigantats la desastrosa calamitat que en tantes poblacions está causant estragos. (El bou, 21) En el mismo sentido, el semanario no se cansará de anunciar el peligro de una nueva epidemia de graves consecuencias económicas, ante la cual muchas familias abandonan la población, con más miedo que razón, y haberse cerrado la mayor parte, o todas las fábricas; que este será el verdadero cólera. (El Bou, 22) La primera respuesta social es una suscripción para socorrer a los enfermos pobres, cuya lista se publica en El Bou, el 30 de agosto, al mismo tiempo que se van dando las cifras de invadidos durante el mes. Los números son menos violentos que durante el brote de 1884, y esta vez la infección permanece en la ciudad menos tiempo, hasta el dichous 17 -de septiembre- [que] según tenim anunsiat se cantà en la Iglesia de Santa María un solemne Te Deum en acsió de grasia per la completa desaparició del cólera. (El Bou, 29) El caso de 1885 es el antepenúltimo episodio de cólera.

El huésped del Gánges regresa durante el verano de 1890. En este momento es El Labrador, dirigido por J. de Mata Coquillat, el semanario que da la voz de alerta de la aparición de la epidemia en Rugat, un municipio de la Vall d'Albaida (Valencia). El periodista vuelve a poner el acento en las consecuencias económicas que puede desencadenar una nueva epidemia, sobre todo para la clase trabajadora, los necesitados, los obreros, no deben ni pueden ser abandonados, de ahí la razón que nos impulsa a dar el primer aviso a nuestras autoridades procurando despejarlo todo de la ficción y la mentira. (El Labrador, 4) Antes incluso de la aparición de infectados, la reacción del Ayuntamiento es activar un nuevo protocolo de 
actuación propuesto por el médico local Alfredo Llopis, que reparte una serie de cartillas sanitarias con las que se pretende informar a la población y con ello combatir los primeros síntomas (Libro de Actas Municipal, 1890). Otra medida, ya habitual, es la suspensión de las fiestas de la Virgen de la Asunción con motivo de la proximidad del cólera en cercanos municipios. (Documento 1) Esta vez la ciudad escapa indemne, también ocurrirá lo mismo en el verano de 1891, (El pueblo, 3) año en que los médicos municipales deciden la inoculación de la vacuna de Ferrán, cuya administración será dirigida el Dr. Campello, que la impone de forma obligatoria de tal modo que los no vacunados no podrán en manera alguna ser admitidos en escuelas, colegios, etc. (El eco liberal, 23)

Durante los años noventa, el cólera irá pasando poco a poco a un segundo plano. La nueva conciencia colectiva de la ciudadanía sobre las condiciones higiénicas, las constantes denuncias de aguas estancadas que pudieran actuar como focos de infección y el nuevo urbanismo de la ciudad contribuyen a superar los ciclos epidémicos, de los que ya sólo tendremos noticia en 1895, cuando la ciudad sufra el último brote importante, en el que se llegan a contabilizar más de 3000 enfermos, la mayoría de los invadidos son pobres que vienen del campo y a quienes no es posible prestar auxilio, ante lo cual, la Junta de Sanidad propone la paralización de la industria y el comercio. (Documento 2). No tenemos constancia de fallecimientos.

\section{LAS CONSECUENCIAS ECONÓMICAS Y SOCIALES, LAS RESPUESTAS DE LA CIUDAD AL CÓLERA: LOS CUIDADOS COLECTIVOS}

Como ya hemos reiterado, la primera consecuencia del cólera en la ciudad es económica. La actividad industrial y comercial se paraliza. Fábricas, talleres y tiendas cierran sus puertas. El tráfico ferroviario queda restringido, y el cordón sanitario reduce a mínimos el contacto con otras ciudades. Entre el 31 de agosto y el 19 de octubre de 1884, Elche se convierte en una ciudad fantasmagórica. Las clases altas y medias, abandonan la ciudad, y miles de trabajadores pierden sus trabajos $\mathrm{y}$ traspasan el umbral de la pobreza. Ya desde que se conoce la epidemia en Marsella, se advierte que, junto al luto, la enfermedad paraliza todos los contratos y transacciones mercantiles. (El Vinalopó, 21) La epidemia afecta gravemente a muchos de los puestos permanentes del mercado municipal. El arrendador de dichos puestos públicos no podrá afrontar la cantidad de la subasta, como consecuencia de la invasión colérica en la misma en 1884 fueron escasos los vendedores de comestibles que se presentaron aquel año y su caudal por ello muchísimo menos [...] por este motivo se le embarga su casa. (Documentos 3, 4) Se produce un cierre masivo de fábricas y talleres, que afecta a más del 70\% de los trabajadores. (Legajo D-83-1). Según el relato de Ildefonso Sansano, por doquier se transitaba, veíanse abandonadas las viviendas: el movimiento había sucedido a la quietud, la expansión al retraimiento, el bullicio a la calma, a la animación la tristeza, a los alegres cantares de las fábricas el sepulcral silencio de las tumbas (El Vinalopó, 36). Como ya hemos dicho, en un alto porcentaje de los casos, el destino de la clase trabajadora es la muerte o la miseria. Por ello, la crisis del cólera morbo activa nuevas estrategias sociales en la ciudad: asociaciones como Juventud Ilicitana, el Círculo Obrero Ilicitano, La misa de once, Círculo Industrial y Sociedad Josefina trazan una conducta humanitaria (El Triángulo, 33), lo cual significa un antes y un después, y sirve como modelo para futuras crisis, de tal modo, que la respuesta de las asociaciones de la ciudad es un rasgo que defina la nueva filantropía de la sociedad ilicitana.

La epidemia se revela como una trance valioso por las respuestas con las que la población reacciona en plena efervescencia de crecimiento económico, hasta el punto de ser un factor configurador del movimiento obrero: al ver cerradas en estos pueblos casi todas las fábricas; sin trabajo y sin pan a muchos obreros enfermos sin socorro, y otras muchas atendibles necesidades (El Triángulo, 26), surge la idea de crear una asociación de socorros mutuos que se materializa en marzo de 1885, en el citado Círculo Obrero Ilicitano, todavía no una organización de clase, aunque pronto actúa como agrupación catalizadora del movimiento obrero local. El periodista Juan de Mata Coquillat insiste en identificar la epidemia como uno de los desencadenantes de la dialéctica entre clase obrera y burguesía local: cerráronse las fábricas, que abandonaron sus dueños, para alejarse del peligro que las amenazaba, sin 
pesar u olvido de no pocos de aquellos que dejaban sin trabajo y sin pan a más de 4.000 obreros (El Labrador, 25).

Y es que la prensa local (Sáez Gómez, 2004) protagoniza una de las respuestas sociales más importantes al dar un seguimiento en primera persona del relato de los hechos. Es el caso de El Vinalopó, que tras el cierre por la epidemia, sale a la calle con la publicación de una carta muy crítica con la actuación del Obispado (El Vinalopó, 31) y continúa con una crónica de 5 artículos, (El Vinalopó, 30-36) en los que se da detallada cuenta del desarrollo de la infección en la ciudad, así como de la actuación política del Ayuntamiento. La prensa trata por primera vez las noticias del huésped del Gánges con una óptica decididamente política y un compromiso con la opinión pública, en el que destaca la labor de los citados Ildefonso Sansano, Juan de Mata Coquillat o José Pérez Sánchez (El Bou, 22, 25, 26).

Por último, el municipio desarrolla una nueva salud pública: intensifica la pavimentación de las calles, construye un nuevo mercado de abastos, remodela su entorno urbano y reforma el plano de la ciudad abriendo espacios mejor ventilados, como la Glorieta del Dr. Campello o el paseo de Cortés, más salubres, y sobre todo, fija un protocolo de actuación que se da a conocer en los bandos municipales y a través de la prensa, en el que participa directamente el personal facultativo (Libro de Actas, 1884) y en el que se vincula al propio pueblo, al que se obliga a rociar de los portales desde 1884 (El Vinalopó, 21,26). Por otro lado, se prohíbe a las fábricas de aguardientes, almidón, jabones el vertido de aguas sucias a acequias de riego o sumideros, se vigila con especial celo el abandono de animales muertos, basuras, estiércol, u otras sustancias de fácil corrupción y se obliga $a$ los dueños $o$ inquilinos el aseo y limpieza de las habitaciones. (El Labrador, 5) La ciudad es capaz de cooperar con los aspectos más sociales de los cuidados colectivos, en especial, se toma conciencia del peligro que suponen las aguas estancadas dentro del municipio: se presta atención a roturas o filtraciones en acequias urbanas (Libro de Actas, 1887), desagües (Legajo D-103), estado de las alcantarillas, depósitos (Documento 5), fuentes públicas (Libro de Actas, 1902), barrizales en la vía pública (El talismán, 5) o fuera de la ciudad, en el pantano o en fincas tan propensas a la inundación como Carrizales (El triángulo, 52/ El pueblo de Elche, 4), en este caso concreto por el riesgo de paludismo. En el siguiente texto se observa la preocupación por ciertas costumbres insalubres: En vista del abuso observado en dejar sueltos por las calles los cerdos, vulgarmente llamados de 'San Antón', costumbre que debe abolirse, si no se quiere sufra resentimiento la cultura de esta población, se acordó prohibirse terminantemente. (Libro de Actas, 1895).

También se restringe la cría, matanza tradicional, venta sin permiso (Documento 6) y consumo de carne durante los meses de calor (El Vinalopó, 21) en la calle de reses de cerda, hechos que, en caso de producirse pueden ser denunciados ante el Ayuntamiento (Documentos 7,8 ). De igual forma se actuará sobre los perros sueltos en la vía pública, sobre todo debido a los horrores de la hidrofobia. (Libro de Actas, 1884)

\section{EPÍLOGO}

En resumen, tras la epidemia de 1884, se dan los primeros pasos en la creación de una mentalidad colectiva en torno a la salud pública; es posible hablar de una sociedad civil que construye una estrategia de cuidados colectivos, por y para la ciudad. El poder y las clases dominantes derivan hacía estrategias claramente biopolíticas: se impone la campaña de vacunación, se obliga a la población a mantener condiciones higiénicas óptimas de cuerpos y objetos. Y, aunque la lucha contra el cólera parte de un presupuesto humanitario, las élites también interpretan la epidemia desde un punto de vista económico, social y de clase. No obstante, la ciudad, tanto en el caso de obreros como el de fabricantes, da una serie de respuestas que calificamos de categorías de cambio social:

En primer lugar, la prensa adquiere un protagonismo crítico y activo, que se convierte en el factor decisivo de cambio social, ya que genera una opinión pública comprometida con la crisis;

En segundo lugar, la respuesta ciudadana -en especial obrera- se traduce en la creación, en marzo de 1885, del Centro Obrero Ilictano, la primera organización protosindical y de socorros mutuos; 
En tercer lugar, la iniciativa municipal se orienta hacia un nuevo urbanismo en la ciudad, en consonancia con el desarrollo de salud pública que se observa en la reforma del mercado central, la apertura de nuevos espacios, la pavimentación de las calles y la prohibición de vertidos -muy frecuentes en una incipiente industria muy desregularizada- que favorecen la ventilación de la ciudad. Entendemos, para finalizar, que la hipótesis propuesta al principio, es un buen punto de partida para la construcción de un modelo de interpretación, ya que

\section{FUENTES Y BIBLIOGRAFÍA}

\section{FUENTES (ARCHIVO HISTÓRICO MUNICIPAL DE ELCHE) (AHME)}

\section{Prensa histórica}

-El Vinalopó, 19-45 (Sig. PH-39) 45-62 (sig. PH84. AHME)

-El bou, 21 (sig.PH-89) 22, 25, 2, 29, 146

(sig.PH-146)

-El talismán, 5 (sig.PH-84)

-El labrador, 4, 5 (sig.PH-9), 25 (sig.PH-81), 89

(sig.PH-89)

-El pueblo, 3 (sig.PH-86)

-El eco liberal, 23 (sig.PH-9)

-El triángulo: 26, 33, 52 (sig.PH-84)

-El pueblo de Elche: 4 (sig.PH-13)

\section{Otros documentos}

Documento 1 (sig. 225-9), Documento 2 (sig. 4.36), Documento 3 (sig. 41-34),

Documento 4 (sig. 41-45), Documento 5 (sig. 35-

2), Documento 6 (sig. 41-52-6),

Documento 7 (sig. 38-59), Documento 8 (sig. 3840)

Legajo (sig. D-83-1)(Fomento)

Legajo (sig. D-103)(Correspondencia) consideramos que estos cambios sociales son categorías fundamentales en el desarrollo de la sociedad civil, la participación/iniciativa ciudadana, y la consolidación de una mentalidad colectiva propia de la contemporaneidad occidental, es decir, las estrategias de grupo generan una nueva complejidad en la idea de cuidado social contra el cólera morbo, este cuidado social será una característica de la sociedad en el siglo XX.

Libro de Actas Municipal:

1890 (sig. a181)(folio 67)

1884 (sig. a 176)(folios 66-77)(folio 63)

1887 (sig. a 178)(folio 173) (folio 14 vuelto)

1902 (sig. a 195)(folio 53v)

1895 (sig. a 186)(folio,110v)

\section{BIBLIOGRAFÍA}

Báguena, Ma. J. (2011). Jaime Ferrán y su papel en las epidemias de cólera de Valencia. Anales (Reial Acadèmia de Medicina de la Comunitat Valenciana), 12, 1-9.

Cardoso, C. (1989). Introducción al trabajo de la investigación histórica. Conocimiento, método e historia, Barcelona: Crítica.

-Herrera, F. (1997). Crisis y Medidas sanitarias en Cádiz (1898-1947). Zaragoza: Cuadernos de Historia de la Ciencia, 10.

Ibarra Ruiz, P. (1895). Historia de Elche. Alicante: Establecimiento tipográfico de Vicente Botella.

Moreno Sáez, Francisco (1987). El Movimiento Obrero en Elche (1890-1931). Alicante: Instituto de Estudios “Juan Gil-Albert”.

Sáez Gómez, J.M.., López González, J., Valera Candel, M., y López Hernández, C. (2004). Medio ambiente, medio social y epidemias: topografía médica de Cartagena y la epidemia de 1885 según Federico Montaldo y Peiró”, Llull. Revista de Historia de las Ciencias y de las Técnicas, 27, 165-190. 